\title{
Kinetics and Gibbs Function Studies on Lipase- Catalyzed Production of Non-Phthalate Plasticizer
}

\author{
Hadeel Hosney ${ }^{1,2 *}$, Eslam G. Al-Sakkari ${ }^{3}$, and Ahmad Mustafa ${ }^{4,5}$ \\ ${ }^{1}$ Chemical Engineering Department, Faculty of Engineering, Minia University, El-Minia, EGYPT \\ ${ }^{2}$ Environmental Engineering program, Zewail City of Science and Technology, Shiekh Ziad District, 6 October, EGYPT \\ ${ }^{3}$ Chemical Engineering Department, Polytechnique Montréal, Montréal, CANADA \\ ${ }^{4}$ Faculty of Engineering, October University for Modern Sciences and Arts (MSA), Giza, EGYPT \\ ${ }^{5}$ Center of Excellence, October University for Modern Sciences and Arts (MSA), Giza, EGYPT
}

\begin{abstract}
Petroleum based phthalate plasticizers encounter enormous claims to prohibit their production due to their harmful health impacts when they are mixed with plastics. That is why efforts are being done to find safer natural alternatives. We have investigated the reaction kinetics of the esterification epoxidation of oleic acid and 2-ethylhexanol in the presence of hydrogen peroxide catalyzed using Candida antarctica lipase (Novozym 435, Novozymes, Kobenhavn, Denmark). The product of this reaction is epoxidized 2-ethylhexyl oleate, a non-phthalate green plasticizer. The kinetic model for this reaction follows a multi-substrate PingPong Bi-Bi mechanism with competitive inhibition by the alcohol. The reaction's kinetic parameters were found to be $0.76 \mathrm{M}, 0.37 \mathrm{M}, 0.08 \mathrm{M}$, and $37.20 \mathrm{mM} / \mathrm{min}$ for Michalis-Menten constant for oleic acid $\left(\mathrm{K}_{\mathrm{mo}}\right)$, Michalis-Menten constant for alcohol $\left(K_{\mathrm{ma}}\right)$, alcohol inhibition constant $\left(K_{\mathrm{ia}}\right)$, and maximum reaction velocity $\left(\mathrm{V}_{\max }\right)$, respectively. Then the Gibbs function analysis of the transition state based on the Arrhenius and Eyring equations was carried out. The internal diffusional limitations were found to be negligible as the effectiveness factor took the value of almost unity. While the external mass transfer resistance had no effect on the reaction due to operating at relatively high agitation speed and high temperature. This investigation confirms that this reaction was only kinetically controlled.
\end{abstract}

Key words: esterification-epoxidation, Novozym 435, plasticizer, Ping-Pong Bi-Bi model, Gibbs function

\section{Introduction}

Poly (vinyl chloride) (PVC) is one of the most predominant polymers in the marketing world due to its low production cost and good mechanical properties. Plasticizers are among the various additives that mixed with PVC during processing. Plasticizers are utilized to impart flexibility and elasticity to the stiff PVC by increasing the spaces between polymer chains and reduce the interactions between them ${ }^{1)}$. Therefore, plasticized PVC finds versatile applications in different areas, such as in medical devices, toys, packing materials, and electronic components.

Di (2-ethylhexyl) or Di octyl phthalate (DEHP or DOP) is considered as the most used primary plasticizer in PVC worldwide; accounting for more than $80 \%$ of the total plasticizers market ${ }^{2}$. However, the European Union (EU) classified DEHP plasticizer as a repro-toxic compound and strongly recommended finding a safer substitute, especially in medical devices ${ }^{3)}$. This was followed by passing the law in France ${ }^{4)}$ to prohibit manufacturing and trading food packaging containing DEHP. This substitution could be achieved by two approaches; the first would be to substitute PVC with another polymer, such as polypropylene or silicone; the other would be to replace phthalate plasticizers by non-phthalate ones ${ }^{1)}$.

Several non-DOP plasticizers have been introduced to the market in recent years; however, some of them are derived from petroleum resources which face gradual depletion. The production of bio-based plasticizers has received much attention in recent decades because they are renewable, biodegradable, and biocompatible ${ }^{5}$. Epoxidized soybean oil, epoxidised sunflower, linseed and palm oils are among the most widely used bio-based non-DOP plasticizers worldwide. In addition, it has been demonstrated that epoxidized fatty acid esters of oleic acid such as di(2-ethylhexyl) oleate (DEHO) possess high levels of compatibility when mixed with PVC owing to their influence on tensile strength, elongation and modulus ${ }^{6)}$.

\footnotetext{
*Correspondence to: Hadeel Hosney, Chemical Engineering Department, Faculty of Engineering, Minia University, El-Minia, EGYPT E-mail: eng.hadeelalaa@gmail.com Accepted March 22, 2020 (received for review January 28, 2020) Journal of Oleo Science ISSN 1345-8957 print / ISSN 1347-3352 online http://www.jstage.jst.go.jp/browse/jos/ http://mc.manusriptcentral.com/jjocs
} 
Over the last decade, the largest ratio of fatty esters/ fatty acids epoxidation processes has been accomplished by the conventional approach of modifying the unsaturated chains through utilizing $\mathrm{H}_{2} \mathrm{SO}_{4}$ and $\mathrm{H}_{3} \mathrm{PO}_{4}$ as catalysts. However, when these acidic conditions are employed, polymerization of fats and by-product formation take place, which generates dark-colored products that need further purification ${ }^{7)}$. In order to achieve such sustainable goals, eco-friendly catalysts with higher selectivity are necessary to be used. The enzymatic alternative biocatalyst showed up as a valid substitute with a greener pathway in comparison to the chemical conventional approach ${ }^{8)}$. As it avoids the undesired side reactions, eliminates the need for a solvent and requires a neutral $\mathrm{pH}$ levels which means that downstream product separation is less costly. Furthermore, it needs less temperature which decreases the thermal demand of the process ${ }^{9,10)}$.

To develop a detailed knowledge about the proposed esterification and epoxidation reactions using biocatalyst and also for the future design of the large-scale process, a kinetic study should be established. According to literature, most of the research studies done on enzymatic kinetics using Novozym 435 as a catalyst were related to fatty acids esterification or triglycerides transesterification reactions. Different models were proposed through these studies. Galgali et al. ${ }^{11)}$ studied the kinetics of citronellol laurate production by ultrasound-assisted esterification using Novozym 435 as a catalyst where it could be found that the experimental data fitted the ordered Bi-Bi kinetic model obtained well. Heeres et al. ${ }^{12)}$ have developed a generic model based on second order reaction kinetics where it proved its ability to fit the observed data and represent the reaction kinetics of the solvent-free lipase-catalyzed production of (meth) acrylate monomers very well. Encinar et $a l .{ }^{13)}$ synthesized biodiesel using Novozym 435 as biocatalysts after sunflower oil transesterification with methanol. Through this investigative study, different mechanisms were proposed to describe reaction kinetics parameters such as Michaelis-Menten, competitive inhibition and Ping-Pong Bi-Bi, and mechanisms ${ }^{13)}$. Another research was carried by Bansode $e t a l .{ }^{14)}$, who used immobilized lipase to achieve an isoamyl butyrate conversion of $96 \%$ under mild operating conditions. The authors found that the esterification reaction followed Ping-Pong Bi-Bi mechanism with alcohol inhibition. Moreover, a kinetic model was set up for enzymatic biodiesel production for castor oil using Novozym, it was found that at higher alcohol to oil molar ration, no inhibition was detected and the reaction followed the Ping-Pong mechanism ${ }^{15)}$.

Although there are many proposed kinetic data and researches for biochemical reactions, there are insufficient data for the synthesis of E-2-EHO as well as a kinetic study of it. Accordingly, this work aimed to study the kinetics and Gibbs function of the production of E-2-EHO through es- terification-epoxidation reaction over Novozym 435. In addition, different possible mass transfer limitations were studied. The kinetic data was used to implement analysis of Gibbs function for the reaction in the transition state. The novelty of this work is that it introduces the kinetic and Gibbs function studies of enzymatic esterification-epoxidation reactions in order of producing green E-2-EHO plasticizer for the first time to the best of our knowledge. From an engineering perspective, this study will help the designers to design a suitable reactor with exact size and dimensions in order to perform the reactions properly.

\section{Material and Methods \\ 2.1 Materials}

Novozym 435 (10,000 U/g; propylarate units PLU) Candida antarctica Lipase B (CALB) in the form of dried powder, physically immobilized by macroporous support, Lewatit VPOC 1600 was provided by Novozymes A/S (Kobenhavn, Denmark). 2-ethyl-1-hexanol $\geq 99 \%$, molecular sieves, $4 \AA$, beads 8-12 mesh, were purchased from Sigma Aldrich (St. louis, MO, USA). Oleic acid 70\% (Gas Chro fatty acids composition: oleic acid $70.38 \%$, palmitic acid $4.32 \%$, stearic acid $6.33 \%$, linoleic acid $18.97 \%$ ), hydrogen peroxide $50 \%$, and sodium hydroxide were purchased from Middle East Co. (Cairo, Egypt). All other reagents were of analytical grade.

\subsection{Kinetic study}

Before conducting the kinetic and Gibbs function studies, the factors affecting the proposed esterification epoxidation reaction were previously studied and optimized. The optimum conditions were found to be a reaction temperature of $65^{\circ} \mathrm{C}$, molar ratio of $\mathrm{H}_{2} \mathrm{O}_{2} / \mathrm{C}=\mathrm{C}$ of fatty acids of $0.5: 1$, alcohol to fatty acid molar ratio of $4: 1$ and enzyme load of $4 \%$ of the mixture weight. Based on the optimization study, all kinetic experiments were conducted at a fixed hydrogen peroxide $\left(\mathrm{H}_{2} \mathrm{O}_{2}\right)$ concentration of 0.5 $\mathrm{mM}$. This concentration was chosen on the basic of avoiding the enzyme inhibition caused by higher concentrations of $\mathrm{H}_{2} \mathrm{O}_{2}$. Also, $\mathrm{H}_{2} \mathrm{O}_{2}$ was added to the reaction mixture stepwise for the same reason of avoiding enzyme inhibition. Stock solutions of oleic acid and 2-ethyl hexanol were prepared with concentrations of 1,2,3 and $4 \mathrm{M}$ by using isobutyl alcohol. The concentrations of 1,2,4 $\mathrm{M}$ for the fatty acid and alcohol were used to develop the kinetic model, while the $3 \mathrm{M}$ concentration was used for testing and validating the model. The effect of the alcohol and oleic acid concentrations on the initial rate of reaction was investigated by fixing the concentration of one substrate at a constant value and varying the concentrations of the other. The analysis of reaction progress and changes in rates were carried by monitoring the depletion of fatty acids titrimetri- 


\section{Kinetic Study}

cally through withdrawing aliquots of reaction mixture each 10 minute as shown in Equation(1).

Reaction Progress $(\%)=\frac{N-N^{\prime}}{N} \times 100$

where:

$N$ : the volume of $\mathrm{NaOH}$ depleted before the reaction.

$N^{\prime}$ : the volume of $\mathrm{NaOH}$ depleted after the reaction.

It should be mentioned here that all experiments were measured in triplicate and the reaction progress values were determined as the mean value of the three measurements.

Reaction rates were calculated by converting the fatty acids conversion values into concentrations which then fed to the numerical differentiation formula developed by Carnahan $e t a l .{ }^{16)}$. The kinetic parameters were estimated graphically using reciprocal analysis of Lineweaver-Burk from the slope of the plots of 2-ethylhexyl alcohol or oleic acid rates versus concentration. Also, all substrates kinetic parameters were analyzed by Ping-Pong Bi-Bi mechanism and then fitted into the reaction rate equation depending on this mechanism. The Ping-Pong Bi-Bi approach is characterized by the transformation of the enzyme into an intermediate form (transition state) when the product is released from the first substrate. The enzyme contained in its original form at the end of the reaction as it is involved in the reaction and is not consumed. Another main feature of the Bi-Bi model is that one product is formed and released before binding with the second substrate.

All experiments were performed in a sealed double jacket reactor at operating volume of $10 \mathrm{~mL}$ and constant operating conditions of $65^{\circ} \mathrm{C}$, molar ratio of $\mathrm{H}_{2} \mathrm{O}_{2} / \mathrm{C}=\mathrm{C}$ of fatty acid 0.5:1, $0.425 \mathrm{~g}$ molecular sieves/g oleic acid, and $200 \mathrm{rpm}$. To characterize the resulted product, $\mathrm{H}^{1} \mathrm{NMR}$ analysis was performed and it was found that both 2-ethyl hexyl oleate ester and epoxidized 2-ethyl hexyl oleate were formed (Figure $\mathbf{S} 1$ is presented in supplementary material section).

\subsection{Gibbs function study}

The activation energy for the proposed enzymatic esterification-epoxidation reaction was estimated by applying the Arrhenius equation using the temperature range between $45^{\circ} \mathrm{C}$ and $65^{\circ} \mathrm{C}$. Equation (2) illustrates the quantitative relationship between activation energy $\left(E_{a}\right)$, rate constant $(k)$, and temperature $(T)$ of assay

$$
\operatorname{Ln}(k)=\ln A-E_{a} \S / R T
$$

where:

$k$ : rate constant $\left(\mathrm{h}^{-1}\right)$,

A: pre-exponential factor $\left(\mathrm{h}^{-1}\right)$,

$E_{a}$ : activation energy $\left(\mathrm{kJ} \mathrm{mol}^{-1}\right)$,

$R$ : ideal gas constant $\left(8.31447 \mathrm{~J} \mathrm{~K}^{-1} \mathrm{~mol}^{-1}\right)$,

$T$ : absolute temperature $(\mathrm{K})$, and

The notation $\S$ refers to the transition state for the enzy- matic reaction.

To evaluate the activation energy at the transition state, a graph of $\mathrm{ln} \mathrm{k}$ versus $1 / \mathrm{T}$ is plotted to obtain the slope $\left(-E_{a} \S / R\right)$ and $\ln A$ is the intercept of this plot. The other parameters, such as activation enthalpy change and activation entropy change were calculated by applying Eyring's activated complex theory $(\mathrm{ACT})^{17)}$, which is described mathematically in Equation(3).

$$
\ln (\mathrm{k} / \mathrm{T})=\left[\ln \left(\mathrm{k}_{\mathrm{B}} / \mathrm{h}\right)+(\Delta \mathrm{S} \S / \mathrm{R})\right]-(\Delta \mathrm{H} \S / \mathrm{R})(1 / \mathrm{T})
$$

where:

$\mathrm{k}_{\mathrm{B}}$ : Boltzmann constant $\left(1.38065 \times 10^{-23} \mathrm{~J} \mathrm{~K}^{-1}\right)$, h: Planck's constant $\left(6.62608 \times 10^{-34} \mathrm{~J} \mathrm{~s}\right)$,

$\Delta \mathrm{H} \S$ : is the enthalpy of activation $\left(\mathrm{kJ} \mathrm{mol}^{-1}\right)$, $\Delta \mathrm{S}$ : entropy of activation $\left(\mathrm{J} \mathrm{mol}^{-1} \mathrm{~K}^{-1}\right)$,

Accordingly, Gibbs free energy can easily be calculated from the Gibbs free energy equation presented in Equation (4).

$$
\Delta \mathrm{G} \S=\Delta \mathrm{H} \S-\mathrm{T} \Delta \mathrm{S} \S
$$

\section{Results and Discussion}

\subsection{Kinetic study}

An optimization study was conducted first before studying the kinetics of the synthesis of E-2-EHO and the reaction variables were analyzed by response surface methodology (RSM). The optimum operation conditions were a temperature of $65^{\circ} \mathrm{C}$, enzyme load of $4 \mathrm{wt} . \%$, alcohol to oleic acid molar ratio of $4: 1$, hydrogen peroxide to $\mathrm{C}=\mathrm{C}$ of fatty acids molar ratio of $0.5: 1$, molecular sieve/g acid of $0.425 \mathrm{~g}$ and reaction time of $2 \mathrm{~h}$. These conditions recorded a maximum experimental fatty acids conversion of $94.2 \%$. Afterword, the kinetic study was performed at the optimum conditions ${ }^{18)}$.

Kinetics experiments were conducted at a constant low hydrogen peroxide concentration, since this showed a significant effect on enzyme inhibition in the optimization study. It was observed that at fixed alcohol and $\mathrm{H}_{2} \mathrm{O}_{2}$ concentrations, the initial rate increases as oleic acid concentration increases. While at fixed oleic acid and $\mathrm{H}_{2} \mathrm{O}_{2}$ concentrations, when the alcohol concentration increases, the initial rate decreases which indicates the inhibition of esterification and epoxidation reactions at higher alcohol concentrations. These observations, are distinctive for enzyme kinetics with competitive inhibition by alcohol at the concentration ranges studied. This comes into agreements with the findings of similar researches that stated that the alcohol has a competitive inhibition pattern over the Novozym 435 at high concentrations ${ }^{19,20)}$. All reaction rates were calculated by applying the numerical differentiation formulas since the data points in the independent variable are equally spaced ${ }^{16)}$. Every result point represents 
the mean from triplicate estimation and the error bars represent the standard deviation.

The kinetics of the enzymatic reaction was analyzed by means of a Lineweaver-Burk approach to estimate the kinetic parameters and to gain an insight into the reaction's mechanism. Figures S2 and S3 illustrate the LineweaverBurk reciprocal plots for the initial reaction rates versus oleic acid concentrations and the initial reaction rates versus alcohol concentrations, respectively at fixed hydrogen peroxide concentration (figures are presented in the supplementary material). The values of determination coefficients $\left(\mathrm{R}^{2}\right)$ were between 0.912 and 0.985 . Figure S1 delineates that the plot profile seems to be parallel for different oleic acid concentrations, indicating that there is no significant inhibition taking place within this concentration range (1, 2 and $4 \mathrm{M})$. These findings were in agreement with what found by Basri et $a l .{ }^{21)}$. The slopes of the reciprocal plot in Fig. S1 are approximately equal, in spite of changing the substrate concentration, which implies that the reaction proceeds through series of binary complex$\mathrm{es}^{20)}$.

Figure S3 illustrates that different lines intersect at 1/ $\mathrm{V}_{\max }$ for higher alcohol concentration; greater than $1 \mathrm{M}$ and the slope for these curves increases with increasing alcohol concentration. These observations are characteristic of alcohol inhibition in two-step transfer reactions, which have been confirmed by plotting the slopes of the intersecting lines against the alcohol concentration, obtaining a straight line as illustrated in Fig. S3. Based on the previous results, it can be stated that the esterification-epoxidation reaction can follow a Ping-Pong Bi-Bi model with alcohol inhibition. Equation (5) can be used to calculate the kinetics parameters based on the proposed model of Ping-Pong $\mathrm{Bi}^{-\mathrm{Bi}^{22)}}$. Results obtained by Cavallaro et $a l .{ }^{23)}$ are consistent with the kinetic results obtained in this research except that the authors reported an inhibition role for the oleic acid, this may be due to using different oleic acid to alcohol molar ratios.

$$
V=\frac{V_{\max } *[O][A]}{K_{m a}[O]+K_{m o}[A]\left\{1+\frac{[A]}{K_{i a}}\right\}+[O][A]}
$$

Where $V$ is the initial rate; $[O]$ and $[A]$ are the concentrations of oleic acid and 2-ethyl hexanol, respectively; $V_{\max }$ is the maximum reaction velocity; $K_{m a}$ and $K_{m o}$ are the Michalis-Menten constants for oleic and 2-ethyl hexanol, respectively; and $K_{i a}$ is the alcohol inhibition constant.

The parameters of the proposed enzymatic reaction kinetics were graphically determined and are tabulated in Table 1. The $K_{m a}$ value estimated for the alcohol is half the $K_{m o}$ value estimated for oleic acid, which indicates the higher affinity of the enzyme to the alcohol than to the acid. $K_{i a}$ was determined graphically by plotting the slopes intersecting line in Fig. S2 versus alcohol concentration ${ }^{20)}$, which is illustrated in Fig. 1. The plot in Fig. 1 resulted in a

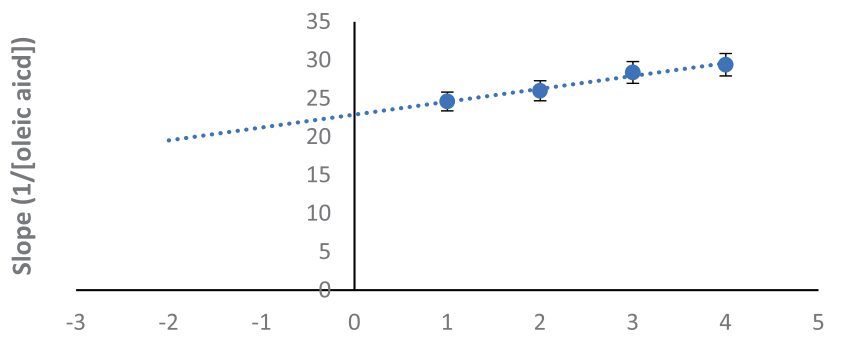

[A] $\mathrm{M}$

Fig. 1 Plot of dissociation constant $\mathrm{K}_{\mathrm{ia}}$ for the enzymatic esterification epoxidation reaction of oleic acid and 2-ethylhexyl alcohol in the presence of hydrogen peroxide.

Table 1 Kinetic parameters for the enzymatic esterification epoxidation reactions of oleic acid and 2-ethylhexyl alcohol, the values with significance level of $\pm 5 \%$.

\begin{tabular}{cr}
\hline Parameter & Value \\
\hline $\mathrm{K}_{\mathrm{mo}} \mathrm{M}$ & 0.76 \\
$\mathrm{~K}_{\mathrm{ma}} \mathrm{M}$ & 0.37 \\
$\mathrm{~K}_{\mathrm{ia}} \mathrm{M}$ & 0.08 \\
$\mathrm{~V}_{\max } \mathrm{mM} / \mathrm{min}$ & 37.20 \\
\hline
\end{tabular}

linear relation which demonstrates one-site inhibition. From Table 1, it can be seen that the value of $K_{m a}$ is approximately four and half times that of $\mathrm{K}_{\mathrm{ia}}$, indicating that the reaction ends with a dead-end inhibition complex. No observed decline in the reaction rate was observed at low alcohol concentration, while the rate increased as the oleic acid concentration increased; thus, supporting the prior claim.

A similar mechanism was proposed for the enzymatic esterification reaction between glycerol and free fatty acids that is present in crude Jatropha oil by immobilized lipase ${ }^{20)}$. Also, the esterification of alanine and glucose catalyzed by Rizomucor miehei lipase and suggested a PingPong Bi-Bi mechanism with competitive inhibition by glucose during this enzymatic reaction ${ }^{24)}$. Liu and Duan ${ }^{25)}$ have established a mathematical model describing transesterification reaction rate of epoxidized soybean oil to form epoxy of methyl ester through using methanol and Novozym 435 as biocatalyst. In this study, they found that the alcohol has an inhibition effect over the enzyme with an alcohol inhibition constant of $0.0004 \mathrm{M}$. This result comes into agreement with the current study in the fact of the similar alcohol inhibition case but with highest obtained value $\left(K_{i a}=0.08\right)$. This may be due to using different alcohol concentrations or it could be because of that 2-ethyl hexyl alcohol is more aggressive over Novozym 435 than the methanol. Chowdary and Prapulla ${ }^{26)}$ found that 


\section{Kinetic Study}

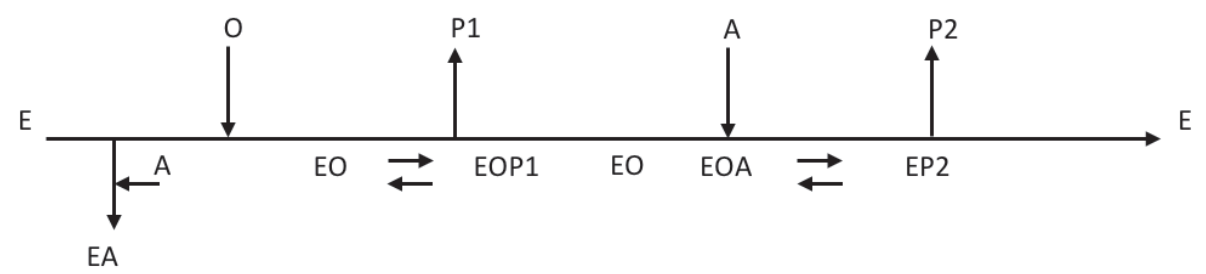

Fig. 2 Schematic diagram of the Ping-Pong Bi-Bi mechanism of lipase-catalyzed epoxidation reaction with competitive inhibition by alcohol[k]. E, enzyme; O, oleic acid; A, Alcohol; P1, water; P2, E-2-EHO; EA), dead-end inhibition complex.

there was an inhibition effect for ethanol over Rhizomucor miehei lipase during the esterification reaction. In their research, it was recorded that the alcohol has an association constant with different values due to using different solvents, where the $\mathrm{K}_{\mathrm{ma}}$ for the alcohol was decreased by $50 \%$ from $0.645 \mathrm{M}$ to $0.256 \mathrm{M}$ when enhancing the solvation. The higher $\mathrm{K}_{\mathrm{ma}}$ value obtained in this research maybe due to using different solvent (isobutyl alcohol), which has different polarity,

The proposed mechanism is illustrated in Fig. 2. Firstly, the enzyme $(\mathrm{E})$ reacts with oleic acid $(\mathrm{O})$ to form an oleicenzyme compound (EO). After isomerization of this compound, water (P1); which is the first product; is released. Then the second substrate, 2-ethyl hexanol (A), is attached to the complex (EO) to form oleic-enzyme-alcohol complex (EOA). The second product (P2) is formed via epoxidation forming an epoxide-enzyme complex (EP2) and then this product is released from the complex regenerating free enzyme (E). The bond formed between the alcohol and the enzyme and then the oleic-enzyme complex is irreversible, leading to the formation of an inactive dead-end complex (EA).

Finally, the validation of the Ping-Pong Bi-Bi model with alcohol inhibition for this reaction was examined by comparing the values of initial rates, obtained after applying the kinetic constants obtained in Table 1 to the proposed model illustrated in Equation(5) for the reaction system, with those obtained experimentally. Figure 3 shows a plot comparing the predicted and experimental values of initial rates, where it can be observed that the coefficient of determination takes a value of 0.994 . These outcomes affirm the conformity of the proposed model with the experimental results.

\subsection{Mass-transfer resistance}

This study examines the resistances of different masstransfer to demonstrate the independence of the reaction from both external and internal diffusion. In addition, it demonstrates that the reaction conditions, as well as the biocatalyst properties, suppressed those mass-transfer resistances. It is worthy of mention that the possible conditions that eliminated external mass transfer resistance, by decreasing the viscosity of the reaction medium hence fa-

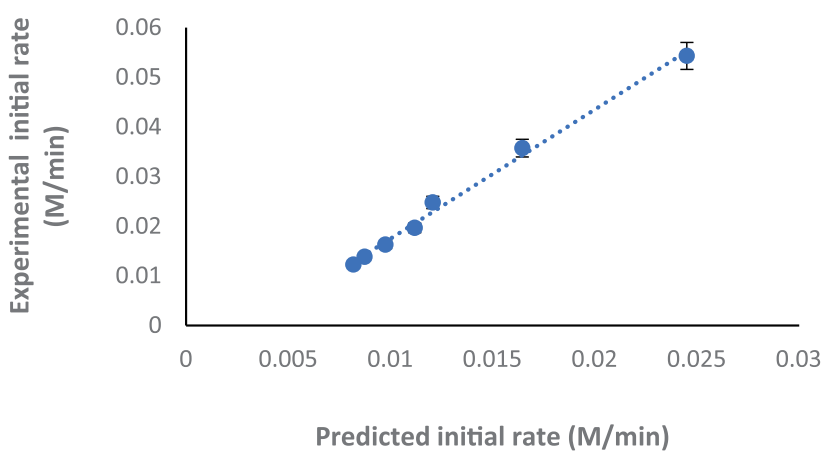

Fig. 3 Predicted versus experimental values of initial rate of reaction for the enzymatic esterification epoxidation reaction of oleic acid and 2-ethylhexly alcohol in the presence of hydrogen peroxide.

Table 2 Reaction mixture average physical properties.

\begin{tabular}{cc}
\hline Property & Value \\
\hline Viscosity, $\mu(\mathrm{Pa} . \mathrm{s})$ & $5.34 * 10^{-3}$ \\
Density, $\rho\left(\mathrm{kg} / \mathrm{m}^{3}\right)$ & 850 \\
Diffusivity (oleic acid in reaction mixture) & $1.9 * 10^{-9}$ \\
$\left(\mathrm{~m}^{2} / \mathrm{s}\right)$ & \\
\hline
\end{tabular}

cilitating the transport and collision of molecules to complete the conversion, are operating at relatively high temperature and using alcohol in excess with lower concentration range that would not have any inhibition effect on the reaction. Moreover, proper agitation improves and maximizes the contact between reactants of different phases inside reaction vessel ${ }^{27)}$. Intra-particle diffusion can be enhanced by using fine biocatalyst particles and thus it is possible to eliminate limitations from mass-transfer.

\subsubsection{External mass-transfer resistance}

The Stocks-Einstein equation was used to calculate the diffusivity presented in Table $2^{28)}$. Further through the reaction, the increasing ester and epoxy concentrations result in decreasing the diffusivity of oleic acid in the mixture; but it is still around the value of $1.9 \times 10^{-9} \mathrm{~m}^{2} / \mathrm{s}$. Epoxidised ester has a very low effect on medium viscosity during the reaction so it can be neglected due to using relatively excess alcohol concentrations.

After determining the acid diffusivity in the reaction 
medium, mass-transfer coefficients can be calculated through Sherwood number which could be determined utilizing the correlation of Frössling, as shown in Equation (6):

$$
S h=2+0.6 * R e^{1 / 2} * S c^{1 / 3}
$$

Prior to employing the Frössling correlation, Schmidt and Reynolds numbers are estimated from Equations (7) and (8) shown below:

$$
\begin{aligned}
& R e=\frac{\rho * d_{p} * U}{\mu} \\
& S c=\frac{\mu}{\rho * D_{A B}}
\end{aligned}
$$

Where, $\rho, \mu, D_{A B}, d_{p}$ and $U$ are average medium density $\left[\mathrm{kg} / \mathrm{m}^{3}\right]$, average medium viscosity $[\mathrm{kg} / \mathrm{m} . \mathrm{s}]$, average oleic acid diffusivity in reaction medium $\left[\mathrm{m}^{2} / \mathrm{s}\right]$, catalyst particle size $[\mathrm{m}]$, medium linear velocity $[\mathrm{m} / \mathrm{s}]$, respectively. Linear velocity $U[\mathrm{~m} / \mathrm{s}]$ can be calculated by multiplying the angular velocity by reactor diameter. Reactions were done under an agitation speed of $200 \mathrm{rpm}$ inside a reactor of an approximate diameter of $0.04 \mathrm{~m}$ while the maximum particle size of catalyst was $0.3 \mathrm{~mm}$, so the linear velocity of the biocatalyst particles was $0.42 \mathrm{~m} / \mathrm{s}$. Using these values and the values in Table 2, Reynolds and Schmidt numbers were calculated to be 20 and 3313.4, respectively. By substituting in the correlation of Frössling, the Sherwood number is calculated to be 42 approximately; hence the mass-transfer coefficient could be obtained using Equation(9). The resultant value of the mass-transfer coefficient $\left(\mathrm{K}_{\mathrm{MT}}\right)$ is about $0.016 \mathrm{~m} / \mathrm{min}$.

$$
S h=\frac{K_{M T} * d_{p}}{D_{A B}}
$$

To ensure that the kinetic study is intrinsic and that no external mass-transfer limitations take place during the reaction, time constants of both reaction $t_{r}$ and diffusion $t_{d}$ should be calculated and compared. Equations (10) and (11) represent the mathematical formulae of these coefficients.

$$
\begin{aligned}
& t_{d}=\frac{D_{A B}}{k_{m}{ }^{2}} \\
& t_{r}=\frac{C_{0}}{V_{o b s}}
\end{aligned}
$$

Where, $V_{\text {obs }}[\mathrm{M} / \mathrm{min}]$ is the observed initial rate of reaction and $C_{0}[\mathrm{M}]$ is the initial concentration of oleic acid. It was found that $t_{d} \ll t_{r}$, as $t_{d}$ and $t_{r}$ take the values of $1 \times 10^{-5}$ and $10 \mathrm{~min}$, respectively, meaning the negligibility of the external resistance of mass transfer is, verifying the earlier hypothesis that the reaction is independent of external diffusion.

The internal mass transfer could be calculated, in order to confirm the total reaction dependence on surface reaction and its independence from mass-transfer resistance.

\subsubsection{Internal mass-transfer resistance}

In this study, internal mass-transfer resistance is dis- cussed to investigate its role as a rate-limiting process compared to the reaction rate on the biocatalyst surface.

MacLeod investigated the relation between the particles size of a catalyst and effectiveness factor ${ }^{29)}$. For example, the effectiveness factor $(\eta)$ for a reaction of transesterification was reached a value close to the unity for particle sizes up to $1 \mathrm{~mm}$ when the oil diffusivity value is $5 \times 10^{-10} \mathrm{~m}^{2} / \mathrm{s}$ (diffusing of oil in methanol). Employing this to our study ( $0.3 \mathrm{~mm}$ of maximum particle size and $1.9 \times 10^{-9} \mathrm{~m}^{2} / \mathrm{s}$ of diffusivity), it can be deduced that the effectiveness factor becomes of unity, indicating the insignificance of the resistance of internal mass-transfer.

Another method to confirm the negligibility of the internal mass transfer resistance is through calculating the Thiele modulus $(\phi)$ and then calculate the effectiveness factor $\eta$ to check whether it is equal to unity. The Thiele modulus takes the form presented in Equation $(12)^{20)}$.

$$
\phi=\frac{V_{o b s}}{D_{A B} * C_{0}} *\left(\frac{\mathrm{R}}{3}\right)^{2}
$$

Where, $V_{\text {obs }}$ is the observed initial rate of reaction, $\mathrm{R}$ is the average radius of catalyst particles and $C_{0}$ is the initial concentration of oleic acid. Substituting the values of each parameter in Eq. 11 gives a Thiele modulus of about 0.1054 , which is a low value. The value of Thiele modulus can then be used in Equation (13) to calculate the effectiveness factor; the value was found to be about 0.9993, implying the negligibility internal mass-transfer resistance.

$$
\eta=\frac{3}{\phi} *\left(\tanh \phi-\frac{1}{\phi}\right)
$$

In other previously performed studies, researchers have studied the effect of mass transfer resistances on the enzymatic esterification reactions and proved that they have negligible contribution on reaction progress. For example, Gofferjé et $a l .{ }^{20)}$ tried to minimize external mass transfer during enzymatic esterification of free fatty acids and glycerol in crude Jatropha oil by using Rhizomucor miehei lipase by operating at relatively higher temperatures such as $60^{\circ} \mathrm{C}$ and stirring rate of $300-400 \mathrm{rpm}$. In addition, it was proved that the reaction did not suffer from internal mass transfer resistances as the calculated effectiveness factor for this reaction system reached unity. Also, Todero et al. ${ }^{30)}$ proved that there are no effects for external and mass transfer resistances on the production of isoamyl butyrate by immobilized TLL and the reaction rates were controlled by the enzyme kinetics. Al-basir et al. ${ }^{31)}$ obtained a mathematical model for biodiesel production from jatropha curcas oil by utilizing enzyme, this model shown that mass transfer resistances can be inhibited by the aid of appropriate agitation levels.

Moreover, in another study where transesterification of glycerides for biodiesel production was conducted in presence of enzymatic catalyst, external and internal diffusional resistances were examined ${ }^{32)}$. In the case of studying external mass transfer, diffusive time (time constant of diffu- 
sion) and kinetic time (reaction time constant) were calculated and compared to each other; where it was found that the kinetic time is much longer than the diffusive one which means that the external diffusional resistance can be neglected. With respect to the internal resistances, it was stated that they did not exist as the enzymes were immobilized on the surface of the support used; hence, the reaction took place only on the catalyst surface without any need to any internal transport of molecules. With respect to the possible mass transfer limitation of free water on Novozym 435, molecular sieves were added to continuously adsorb the waters in the aqueous phase. Dang et al. reported that the decrees of water content in the medium would lead to increased percentage of conversion in the esterification reaction of oleic acid and fructose catalyzed by Rhizomucor miehei lipase ${ }^{33)}$.

\subsection{Gibbs function study}

Activation energies for esterification epoxidation reaction for the production of 2-ethylhexyl oleate was estimated from the Arrhenius plot as shown in Fig. 4, which yields straight lines with determination coefficients $\left(R^{2}\right)$ varying in the range 0.975-0.999. For Michaelis-Menten constants for oleic acid, alcohol and maximum reaction rate, the increasing trends are observed up at $65^{\circ} \mathrm{C}$, but at $70^{\circ} \mathrm{C}$ they decrease, indicating the possible thermal deactivation of the enzyme at higher temperatures. Similar values were obtained when used Novozym435 from Aspergillus niger source as catalyst in the process of esterification of oleic acid. In the present study, the rate equation constants at different temperatures from 318 to $338 \mathrm{~K}$ were obtained with enzyme loading 4 wt.\%, molar ratio 2-ethyl hexanol/ oleic acid 4:1 and $\mathrm{H}_{2} \mathrm{O}_{2}$ /oleic acid 0.5:1, 0.425 g molecular sieve/g oleic acid, and stirring at $200 \mathrm{rpm}^{34)}$. The activation energies $\left(E_{a} \S\right)$ calculated were $20.45,50.45,17.6$ and 63.13 $\mathrm{kJ} / \mathrm{mol}$ for oleic acid reaction, alcohol reaction, alcohol inhibition reaction, and maximum reaction rate, respectively. The previous findings clearly indicated that the inhibition reaction by alcohol has the lowest activation energy, which means that it is the easiest reaction taking place among these reactions. This confirms the results obtained from kinetic studies that there is an inhibition taking place due to alcohol.

As mentioned before, parameters $(\Delta H \S$ and $\Delta S \S)$ were determined by using the activated complex theory (ACT), described in Equation $(3)^{35)}$. Figure 4 displays the linear regression of the data obtained by Equation(3) for different associated reactions in the esterification-epoxidation reaction, which matches the expectation for a single rate-limited thermally activated process ${ }^{36)}$. It was found that the value of $\Delta \mathrm{H}$ is positive; this can be attributed to the endothermic nature of the lipase catalyzed reactions ${ }^{36-38)}$. As the reaction proceeds, the enzyme-substrate complex concentration increases due to consumption of substrates. Ac-

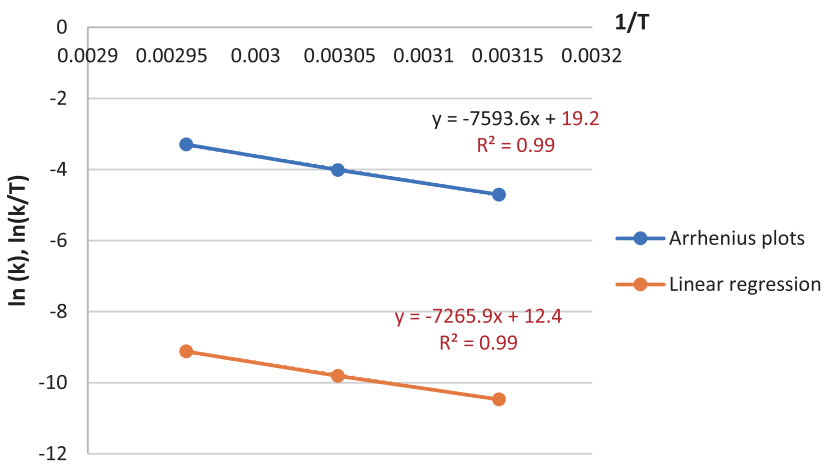

Fig. 4 Arrhenius plot and the Linear regression of $\ln k / T$ as a function of temperature for esterification epoxidation reaction of 2-ethylhexyl ester catalyzed by Novozym 435 at enzyme loading 4 wt. \%, molar ratios of 2-ethyl hexanol/oleic acid 4:1, and $\mathrm{H}_{2} \mathrm{O}_{2} / \mathrm{C}=\mathrm{C}$ of fatty acids of $0.5: 1,0.425 \mathrm{~g}$ molecular sieve/g oleic acid, and stirring at $200 \mathrm{rpm}$.

Table 3 Parameters for the transition state of enzymatic esterification epoxidation reaction for epoxidised 2-ethylhexyl oleate, the values with significance level of $\pm 5 \%$.

\begin{tabular}{cccc}
\hline \multirow{2}{*}{ Parameters } & \multicolumn{3}{c}{ Temperature $(\mathrm{K})$} \\
\cline { 2 - 4 } & 318 & 328 & 338 \\
\hline $\mathrm{k}$ & 0.009 & 0.018 & 0.037 \\
Activation Energy $(\mathrm{kJ} / \mathrm{mol})$ & & 63.1 & \\
Enthalpy change $(\mathrm{kJ} / \mathrm{mol})$ & \multicolumn{3}{c}{60.4} \\
Entropy change $(\mathrm{kJ} / \mathrm{mol} . \mathrm{K})$ & \multicolumn{3}{c}{0.095} \\
Gibbs Function $(\mathrm{kJ} / \mathrm{mol})$ & 90.5 & 91.5 & 92.4 \\
\hline
\end{tabular}

cordingly, entropy change was found to have a negative value which means non-spontaneous complex formation ${ }^{38)}$. Negative entropy change is common for adsorption of molecules onto the surface of solid catalyst or adsorbent and in the case of polymerization as well. It is possible to calculate Gibbs free energy $(\Delta G \S)$ from the enthalpy and entropy change of the transition state with the aid of the Gibbs free energy equation as shown in Equation(4). All parameters are shown in Table 3, which illustrates the effect of temperature changes on Gibbs free energy $(\Delta \mathrm{G})$ for the studied reaction. The values of $(\Delta \mathrm{G})$ were slightly affected and were found to be around $90.5,91.5,92.4 \mathrm{~kJ} / \mathrm{mol}$ at 318, 328, 338 $\mathrm{K}$, respectively. It should be noticed that all the $(\Delta \mathrm{G})$ values are positive which assures the non-spontaneous nature of the esterification-epoxidation process.

\section{Conclusion}

In this paper, the kinetics of enzymatic esterification-epoxidation of oleic acid and 2-ethyl hexanol in the presence 
of hydrogen peroxide using Novozym 435 as biocatalyst was studied. Reactions were performed at operating volume of $10 \mathrm{~mL}$ and constant enzyme loading, hydrogen peroxide to carbon double bond ratio and agitation speed; while the reaction temperatures varied from 318 to $338 \mathrm{~K}$. The kinetics of this enzymatic reaction was found to follow a Ping-Pong Bi-Bi model with alcohol inhibition. After applying this model and determination its parameters as well as comparing its predicted values with the experimental ones, it was found to be an adequate representative of the enzymatic esterification-epoxidation reaction as the determination coefficient took the value of 0.994. In addition, it was found that the reaction is not limited by diffusion, after examining both external and internal mass-transfer resistances, where the reaction was found to be only kinetically controlled. The analysis of Gibbs function confirmed the endothermic nature of the reaction, as the enthalpy of activation take positive value. In addition, the entropy changes were found to take negative values; this is consistent with the fact that the formation of epoxy molecules from oleic acid and alcohol ester molecules and adsorption of both acid and alcohol molecules on the enzyme surface are followed by decreases of entropy and freedom.

\section{Conflict of Interest}

The authors declare that they have no conflict of interest.

\section{Supporting Information}

This material is available free of charge via the Internet at http://dx.doi.org/jos.69.10.5650/jos.ess20025

\section{References}

1) Hosney, H.; Nadiem, B.; Ashour, I.; Mustafa, I.; ElShibiny, A. Epoxidized vegetable oil and bio - based materials as PVC plasticizer. J. Appl. Polym. Sci. 135, 46270 (2018).

2) Samarth, N.B.; Mahanwar, P.A. Modified vegetable oil based additives as a future polymeric material - Review. Open J. Org. Polym. Mater. 5, 1-22(2015).

3) Regulation(EC) No 1272/2008 of the European Parliament and of the council. OJEU. (2008).

4) LOI no. 2012-1442. OJFR. (2012).

5) Borsato, D.; Galvan, D.; Pereira, J.L.; Orives, J.R.; Angilelli, K.G.; Coppo, R.L. Kinetic and thermodynamic parameters of biodiesel oxidation with synthetic antioxidants: Simplex centroid mixture design. J. Braz. Chem. Soc. 25, 1984-1992(2014).
6) Omrani, I.; Ahmadi, A.; Farhadian, A.; Shendi, H.K.; Babanejad, N.; Nabid, M.R. Synthesis of a bio-based plasticiser from oleic acid and its evaluation in PVC formulations. J. Polym. Test. 56, 237-244(2016).

7) Hosney, H.; Mustafa, A. Semi-continuous production of 2-ethyl hexyl ester in a packed bed reactor: Optimization and economic evaluation. J. Oleo Sci. 69, 31-41 (2020).

8) Abdelmoez, W.; Mostafa, N.A.; Mustafa, A. Utilization of oleochemical industry residues as substrates for lipase production for enzymatic sunflower oil hydrolysis. J. Clean. Prod. 59, 290-297 (2013).

9) Mustafa, A.; Karmali, A.; Abdelmoez, W. Optimization and economic assessment of lipase-catalysed production of monoesters using Rhizomucor miehei lipase in a solvent-free system. J. Clean. Prod. 137, 953-964 (2016).

10) Abdelmoezl, W.; Mustafa, A. Oleochemicals industry future through biotechnology. J. Oleo Sci. 63, 545-554 (2014).

11) Galgali, A.; Gawas, S.D.; Rathod, V.K. Ultrasound assisted synthesis of citronellol laurate by using Novozym 435. Catal. Today 309, 133-139(2018).

12) Heeres, A.; Vanbroekhoven, K.; Hecke, W.V. Solventfree lipase-catalyzed production of (meth) acrylate monomers: Experimental results and kinetic modeling. Biochem. Eng. J. 142, 162-169(2019).

13) Encinar, J.M.; González, J.F.; Sánchez, N.; Delgado, S.N. Sunflower oil transesterification with methanol using immobilized lipase enzymes. Bioproc. Biosyst. Eng. 42, 157-166 (2019).

14) Bansode, S.R.; Hardikar, M.A.; Rathod, V.K. Evaluation of reaction parameters and kinetic modelling for Novozym 435 catalyzed synthesis of isoamyl butyrate. $J$. Chem. Technol. Biotechnol. 92, 1306-1314(2017).

15) Zarejousheghani, F.; Kariminia, H.-R.; Khorasheh, F. Kinetic modelling of enzymatic biodiesel production from castor oil: Temperature dependence of the Ping Pong parameters. Can. J. Chem. Eng. 94, 512-517 (2016).

16) Carnahan, B.; Luther, H.A.; Wilke, J.O. Applied numerical methods. Wiley, New York(1969).

17) Eyring, H. The activated complex in chemical reactions. J. Chem. Phys. 3, 107-115(1935).

18) Hosney, H.; AL-Sakkari, E.G.; Mustafa, A.; Ashour, I.; Mustafa, I.; El-Shibiny, A. A cleaner enzymatic approach for producing non-phthalate plasticiser to replace toxic-based phthalates. Clean Technol. Environ. Policy 22, 73-89(2020).

19) Ferrao-Gonzales, A.D.; Veras, I.C.; Silva, F.A.L.; Alvarez, H.M.; Moreau, V.H. Thermodynamic analysis of the kinetics reactions of the production of FAME and FAEE using Novozyme 435 as catalyst. Fuel Process Technol. 92, 1007-1011 (2011). 
20) Gofferje, G.; Stabler, A.; Herfellner, T.; SchweiggertWeisz, U.; Floter, E. Kinetics of enzymatic esterification of glycerol and free fatty acids in crude Jatropha oil by immobilized lipase from Rhizomucor miehei. J. Mol. Catal. 107, 1-7(2014).

21) Basri, M.; Kassim, M.A.; Mohamed, R.; Ariff, A. Optimization and kinetic study on the synthesis of palm oil ester using Lipozyme TL IM. J. Mol. Catal. 85, 214219 (2013).

22) Bansode, S.R.; Hardikar, M.A.; Rathod, V.K. Evaluation of reaction parameters and kinetic modelling for Novozym 435 catalyzed synthesis of isoamyl butyrate. J. Chem. Technol. Biotechnol. 92, 1306 -1314(2017).

23) Cavallaro, V.; Tonetto, G.; Ferreira, M.L. Optimization of the enzymatic synthesis of pentyl oleate with lipase immobilized onto novel structured support. Fermentation 5 (2), 48(2019).

24) Somashekar, B.R.; Lohith, K.; Manohar, K.; Divakar, S. inhibition of Rhizomucor miehei and Candida rugo$s a$ lipases by D-glucose in esterification between L-alanine and D-glucose. J. Biosci. Bioeng. 103, 122-128 (2007).

25) Liu, W.; Duan, F. Lipase-catalyzed transesterification of epoxidized soybean oil to prepare epoxy methyl esters. Grasasy Aceites 69, 247-256 (2018).

26) Chowdary, G.V.; Prapulla, S.G. Kinetic study on lipasecatalyzed esterification in organic solvents. Indian $J$. Chem. B 44B, 2322-2327 (2005).

27) Sim, J.H.; Harun, K.A.; Bhatia, S. Effect of mass transfer and enzyme loading on the biodiesel yield and reaction rate in the enzymatic transesterification of crude palm oil. Energy Fuels 23, 4651-4659 (2009).

28) Geankoplis, C.J. Transport processes and unit operations. $3^{\text {rd }}$ ed., Prentice-Hall International Inc., USA (1993).

29) Macleod, C. Ph.D. thesis. Newcastle University, School of Chemical Engineering and Advanced Materials, Newcastle, UK(2008).
30) Todero, L.M.; Bassi, J.J.; Lage, F.A.P.; Corradini, M. C.C.; Barboza, J.C.S.; Hirata, D.B.; Mendes, A.A. Enzymatic synthesis of isoamyl butyrate catalyzed by immobilized lipase on poly-methacrylate particles: Optimization, reusability and mass transfer studies. Bioprocess Biosyst. Eng. 38, 1601-16013(2015).

31) Al Basir, F.; Datta, S.; Roy, P.K. Studies on biodiesel production from Jatropha curcas oil using chemical and biochemical methods-a mathematical approach. Fuel 158, 503-511 (2015).

32) Bansode, S.R.; Hardikar, M.A.; Rathod, V.K. Evaluation of reaction parameters and kinetic modelling for Novozym 435 catalyzed synthesis of isoamyl butyrate. $J$. Chem. Technol. Biotechnol. 92, 1306-1314(2017).

33) Calabro, V.; Ricca, E.; De Paola, M.G.; Curcio, S.; Iorio, G. Kinetics of enzymatic trans-esterification of glycerides for biodiesel production, Bioprocess Biosyst. Eng. 33, 701-710(2010).

34) Dang, H.T.; Obiri, O.; Hayes, D.G. Feed batch addition of saccharide during saccharide-fatty acid esterification catalyzed by immobilized lipase: Time course, water activity, and kinetic model. J. Am. Oil Chem. Soc. 82, 487-493 (2005).

35) Mahmud, M.S.; Safinski, T.; Nelson, M.I.; Adesina, A.A. Enhancement of heterogeneous ethyl oleate synthesis using hydrous ethanol. Aust. J. Basic Appl. Sci. 11 (3), 160-164(2017).

36) Ferreira, H.V.; Rocha, L.C.; Severino, R.P.; Viana, R.B.; Silva, A.B.F.; Porto, A.L.M. Enzymatic resolution of racemic sulcatol by lipase from Candida antarctica in a large scale. J. Iran Chem. Soc. 7, 883-889 (2010).

37) Gohri, M.I.; Iqbal, M.J.; Hameed, A. Characterization of a novel lipase from Bacillus sp. isolated from tannery wastes. Braz. J. Microbiol. 42, 22-29 (2011).

38) Sharma, A.; Dalai, A.K.; Chaurasia, S.P. Thermodynamic study of hydrolysis and esterification reactions with immobilized lipases. Eur. Int. J. Sci. Technol. 4, 128-136 (2015). 